
Pathophysiology of Haemostasis and Thrombosis

\title{
Treatment with Vitamin K Antagonists: Frequency of Indications and Appropriateness of Continuation
}

\author{
J .F. van der Heijden ${ }^{a, b}$ M.G.H. Remkes ${ }^{c}$ H.R. Büller ${ }^{b}$ M. Vermeulend \\ aLaboratory for Experimental Internal Medicine, bLaboratory for Vascular Medicine, cThrombosis Service \\ Amsterdam, and dDepartment of Neurology, Academic Medical Center, University of Amsterdam, \\ Amsterdam, The Netherlands
}

\section{Key Words \\ Vitamin K antagonist · Indication · Bleeding}

\begin{abstract}
To prevent venous and arterial thrombosis vitamin $\mathrm{K}$ antagonists (VKA) are the treatment of choice for many indications. It is important to balance the benefits and the potential hazards of this treatment. An effective way to prevent unnecessary bleeding during VKA treatment is to stop treatment when the indication is no longer present. In this study, we analyze the distribution of indications in a randomly selected group of 250 patients starting VKA treatment at the Amsterdam Thrombosis Service. The proportion of patients still treated after one year of follow-up was also investigated. The distribution of the indications among patients starting VKA therapy was approximately $50 \%$ for venous thromboembolism treatment and prophylaxis, and approximately $50 \%$ for prophylaxis of arterial thrombosis. After one year of follow-up, 164 (65.6\%) of the 250 patients had stopped VKA therapy. Reasons for stopping included: no indication for continuing VKA treatment, e.g. end of treatment, prophylaxis or restoration of sinus rhythm (137 patients); death (17) and other reasons (10). Six (2.4\%; 95\% Cl: 0.9-5.1\%)
\end{abstract}

patients had a questionable indication for long-term treatment, and 9 (3.6\%, 95\% Cl: 1.6-6.7\%) patients had no clear indication for continued VKA treatment. We conclude that in the setting of the Amsterdam Thrombosis Service, only a small proportion of patients is treated with long-term VKA therapy without a valid indication after 1 year.

Copyright @2002 S. KargerAG, Basel

\section{Introduction}

In the last decade, the number of indications for vitamin $\mathrm{K}$ antagonist (VKA) therapy has increased considerably. VKAs have been used for years in the treatment of venous thromboembolism (VTE) to prevent recurrent disease [1] and arterial thrombosis in patients with mechanical heart valves [2]. For more recent indications, evidence of the beneficial effects of VKAs has been gathered in different large clinical trials and includes atrial fibrillation [3], with or without stroke [4], and postdischarge prophylaxis for VTE after major orthopedic surgery [5]. Another reason for the increase in the number of patients receiving VKAs is aging in the general population.

\section{KARGER \\ Fax +41613061234 \\ E-Mail karger@karger.ch \\ www.karger.com

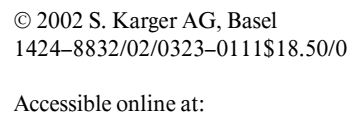

J.F. van der Heijden, MD

Academic Medical Center

Laboratory for Experimental Internal Medicine (Room F4-121), PO Box 22660

NL-1100 DD Amsterdam (The Netherlands)

Tel. +3120566 9111, Fax +3120697 7192, E-Mail j.f.vanderheijden@amc.uva.nl 
Since there exists a delicate balance between preventing thrombosis with VKAs and the risk of bleeding, controlling the level of anticoagulation is of great importance [6]. This is best achieved by measuring the International Normalized Raito (INR) at regular intervals. Despite regular control of the intensity of VKA treatment, the annual incidences of major and minor hemorrhages are approximately 3 and $17 \%$, respectively [7]. Hence, in order to prevent unnecessary harm, it is important to check regularly, whether the indication is still valid and whether the benefit/risk ratio of the VKA therapy is still positive.

In the Netherlands, approximately $1 \%$ of the population is treated with VKAs. Most of these patients are monitored by regional thrombosis services, which form a network covering the entire country. It is common practice for treating physicians and general practitioners to refer patients to these institutes for the management of VKA treatment.

This service is widely appreciated and has several advantages in terms of logistics, quality control and costeffectiveness. However, the major disadvantage may be that patients treated with VKAs do not stop treatment at the appropriate time. Furthermore, at this time the distribution of the indications of VKA treatment among patients initiating this therapy is unknown.

We therefore randomly selected consecutive patients starting with VKAs referred to a thrombosis service. We listed the primary indications and followed these patients for at least one year to assess the frequency and appropriateness of continued VKA therapy.

\section{Patients and Methods}

The study was conducted at the Thrombosis Service Amsterdam, The Netherlands, which provides management of VKA treatment for the larger Amsterdam region. From May 2000 to June 2000 we identified a total of 730 consecutive patients starting VKA therapy. A random number of 250 patients were selected from this cohort.

The following information was recorded: Age, sex, indication(s) for VKA treatment, starting date, aimed stopping date, and target INR. The primary indication for VKAs was determined with the information provided by the referring physician. The physicians in the Thrombosis Service did not know which patients were included in the study; this approach was chosen in order to study the normal care provided by the Thrombosis Service.

All patients were followed for at least one year or until the VKA treatment was stopped. In those patients continuing to receive VKA therapy, the indication for continued use was evaluated by an independent committee. We used three categories for this evaluation: 'definite indication', e.g. mechanical heart valves; 'questionable indication', e.g. peripheral vascular disease or prior myocardial infarction; and 'no clear indication' for long-term VKA therapy, e.g. prophylaxis or treatment of VTE. For the patients who stopped treatment with VKAs during the follow-up period, reasons were recorded and categorized. We used the following categories: 'no indication for continuing VKA treatment', e.g. end of treatment, prophylaxis or restoration of sinus rhythm; 'deceased'; 'bleeding complications' and 'other reasons'.

Major bleeding was defined as overt bleeding associated with a decrease of $2 \mathrm{~g} / \mathrm{dl}$ or more in the hemoglobin level, the need for hospitalization and a blood transfusion of 2 units or more, and that it was intracranial, retroperitoneal, intramuscular or intraarticular. All others were classified as minor. Whenever death occurred, we evaluated whether or not the patient died of a cause directly related to the VKA treatment.

\section{Results}

A total of 250 consecutive patients starting VKA treatment were identified. The mean age was 67 years $(\mathrm{SD} \pm$ 17 , range: $17-91$ years), $46 \%$ were males, and $98 \%$ of the patients used acenocoumarol. The median follow-up was 140 days (range 3-365 days). Two hundred and eighteen (87\%) patients had a single indication for VKA treatment, $30(12 \%)$ had two indications, whereas $2(0.8 \%)$ patients had three. The first of these two patients had atrial fibrillation with a recent myocardial infarction and angina pectoris; the second had heart failure with a thrombus in the heart and pulmonary embolism.

Table 1 summarizes the primary indications for VKA treatment for all patients. The 2 most frequent indications were prophylaxis of VTE and chronic atrial fibrillation, accounting for approximately $60 \%$ of all indications. After one year of follow-up, $164(65.6 \%)$ of the 250 patients had stopped VKA treatment (table 2). Reasons for stopping included: no indication for continuing VKA treatment, e.g. end of treatment, prophylaxis or restoration of sinus rhythm (137 patients), deceased (17) and other reasons (10). None of these patients stopped treatment because of hemorrhage. Six patients experienced minor bleeding complications, but no major bleeding complications were observed. Eighty-six (34.4\%) patients were still using VKAs after the total follow-up period of one year. Of these 86 patients, 71 had a definite indication for longterm VKA treatment, including mechanical heart valves and chronic atrial fibrillation. Six $(2.4 \%$; $95 \%$ CI: $0.9-$ $5.1 \%)$ patients had a questionable indication for longterm VKA treatment, and 9 patients had no clear indication for continued VKA treatment $(3.6 \%, 95 \%$ CI: $1.6-$ $6.7 \%$ ). Three of the 65 patients with a questionable indication were treated for peripheral arterial disease, 2 for cerebrovascular indications, and the indication of the 6 th patient was coronary artery disease. In 8 patients without 
Table 1. Distribution of primary indications for VKA therapy at the start of treatment

\begin{tabular}{lcc}
\hline Indication & Patients & $\%$ \\
\hline Chronic AF & 60 & 24 \\
Cardioversion for AF & 13 & 5.2 \\
Mechanical heart valves & 9 & 3.6 \\
Recent CABG or PTCA & 8 & 3.2 \\
Ischemic heart disease & 1 & 0.4 \\
Other cardiac indications & 11 & 4.2 \\
\hline AF with ischemic stroke or TIA & 6 & 2.4 \\
Recent ischemic stroke & 3 & 1.2 \\
\hline Peripheral arterial bypass surgery & 7 & 2.8 \\
\hline VTE treatment & 38 & 15.2 \\
Prophylaxis of venous thromboembolism & 94 & 37.6 \\
\hline Total & 250 & 100 \\
\hline
\end{tabular}

$\mathrm{AF}=$ Atrial fibrillation; $\mathrm{CABG}=$ coronary artery bypass graft; PTCA = percutaneous transluminal coronary angioplasty; TIA = transient ischemic attack; VTE = deep venous thrombosis or pulmonary embolism.

clear indication, the indication was treatment of VTE, while the remaining patient continued VKA treatment for prophylaxis of VTE after prolonged immobilization.

\section{Discussion}

This study of a randomly selected group of patients starting VKA treatment and managed by the Thrombosis Service Amsterdam shows that 3.6\% (95\% CI: 1.6-6.7\%) of these patients were still using VKAs after one year without a clear indication for long-term use. To our knowledge, this is the first time the proportion of patients still treated with VKAs during follow-up is reported. Several large cohort studies have been conducted in patients starting VKA, but these studies usually focussed on the incidence of hemorrhagic and thrombotic complications. The observed distribution of indications in our study is comparable to that seen in other cohort studies [8].

The present study was conducted in the Thrombosis Service of the greater Amsterdam region. In the Netherlands, patients treated with VKAs are managed by similar services. We therefore feel that these results may be extrapolated to the rest of the country. Although this organization is not unique to this country but also in operation in Italy and parts of the UK, extrapolation on a wide scale
Table 2. Treatment status of the 250 patients after one year of follow-up

\begin{tabular}{lcc}
\hline & Patients & $\%$ \\
\hline Patients who had stopped VKAs & 164 & 65.6 \\
Still treated with VKA & 86 & 34.4 \\
Still using VKAs with a valid indication & 71 & 28 \\
Still using VKAs with questionable indication & 6 & 2.4 \\
Still using VKAs without a clear indication & & \\
$\quad$ for long-term treatment & 9 & 3.6 \\
\hline
\end{tabular}

should be done with caution. In the Netherlands, the treating physicians refer the patients to their regional thrombosis service and thereby delegate the management. They complete a registration form, which details the major indication(s), the desired target range and expected duration of therapy. It is therefore conceivable that patients are still receiving VKAs without a valid indication for prolonged use. However, our findings indicate that this only applies to a small subset of less than $5 \%$ of those followed for one year.

The major limitation of our study is that we completely relied on the information provided at entry by the treating physician and the information collected by the Thrombosis Service during follow-up of the patients. The nature of the surveillance is that patients are being managed for their VKA treatment, but the Service is not always aware of a change in the medical situation of an individual patient and has to obey privacy rules. Therefore, we could not provide precise estimates as to whether the treatment with VKAs actually should have been stopped in all identified patients with a questionable or no clear indication. It is, for example, possible that during the treatment with VKAs a number of patients developed other indications for VKA treatment. Furthermore, we censored our observations by one year, however, when checking the database after another 3 months another 4 patients without a clear indication had stopped VKA treatment following the instructions of the treating physician. All these patients had been treated for VTE. For these reasons, the number reported is probably lower than the $3.6 \%$ observed. On the other hand, as previously mentioned, there will always be a subgroup of patients in whom due to a lack of solid clinical evidence, the continuation of VKA treatment remains subject to discussion; the present debate whether a treatment for 6-12 months is more appropriate for VTE is a good example [9]. The follow-up of our study ended before the publication of the study by Angelli et al. [9]; 
furthermore, during the follow-up of our study, the consensus here in the Netherlands was to treat patients with a first episode of VTE for 3-6 months. However, considering that treatment with VKAs for one year is possibly appropriate for this indication, the number of patients with no clear indication for prolonged treatment with VKAs would be 16 patients $(6.4 \%$; $95 \%$ CI: $3.7-10.2 \%)$, and only 1 patient $(0.4 \%, 95 \%$ CI: $0.0-2.2 \%)$ would receive VKA treatment without a clear indication.

It is of interest to note that, although the percentage of patients still treated after one year without good reason is low, extrapolating this percentage to all patients treated with VKAs in Amsterdam reveals that approximately 600 patients are exposed to the risk of unnecessary anticoagulation. When assuming that the annual risk of serious bleeding is approximately 3\% [7], this totals 18 major bleedings each year.

What can be done to avoid this unnecessary continuation of VKA treatment? Firstly, it would be important for treating physicians to develop a system enabling them to check at regular intervals whether the indication for continued VKA therapy is still present. Secondly, more direct contact between the thrombosis service and the treating physicians would be welcome whenever unnecessary anticoagulation is suspected by the service. This should possibly be implemented by a quality control system, which is best set up in cooperation between referring specialists and the thrombosis service. Finally, the adequate duration of VKA therapy for certain indications, e.g. VTE [9] and atrial fibrillation, is currently the cause of an intense debate. More adequately designed trials are needed to clarify these points.

In conclusion, we found that half of the patients starting VKA therapy are treated for venous indications, while the other half received VKAs as a prophylaxis against arterial thrombosis. Furthermore, we found only a small proportion of patients treated with long-term VKA therapy without a clear indication for this treatment.

\section{References}

1 Hyers TM, Agnelli G, Hull RD, Morris TA, Samama M, Tapson V, Weg JG: Antithrombotic therapy for venous thromboembolic disease. Chest 2001;119(suppl 1):176S-193S.

2 Stein PD, Alpert JS, Bussey HI, Daler JE, Turpie AG: Antithrombotic therapy in patients with mechanical and biological prosthetic heart valves. Chest 2001;119 (suppl 1):220S-227S.

3 Albers GW, Dalen JE, Laupacis A, Manning WJ, Petersen P, Singer DE: Antithrombotic therapy in atrial fibrillation. Chest 2001; 119(suppl 1): 194S-206S.

4 Albers GW, Amarenco P, Easton JD, Sacco RL, Teal P: Antithrombotic and thrombolytic therapy for ischemic stroke. Chest 2001;119 (suppl 1):300S-320S.
5 Geerts WH, Heit JA, Clagett GP, Pineo GF, Colwell CW, Anderson FA Jr, Wheeler HB: Prevention of venous thromboembolism. Chest 2001;119(suppl 1):132S-175S.

6 Cannegieter SC, Rosendaal FR, Wintzen AR, van der Meer FJ, Vandenbroucke JP, Briet E: Optimal oral anticoagulant therapy in patients with mechanical heart valves. N Engl J Med 1995;333:11-17.

7 van der Meer FJ, Rosendaal FR, Vandenbroucke JP, Briet E: Bleeding complications in oral anticoagulant therapy. An analysis of risk factors. Arch Intern Med 1993;153:15571562.
8 Palareti G, Leali N, Coccheri S, Poggi M, Manotti C, Angelo AD, Pengo V, Erba N, Moia M, Ciavarella N, Devoto G, Berrettini M, Musolesi S: Bleeding complications of oral anticoagulant treatment: An inception-cohort, prospective collaborative study (ISCOAT). Italian Study on Complication of Oral Anticoagulant Therapy. Lancet 1996;348:423-428.

9 Agnelli G, Prandoni P, Santamaria MG, Bagatella P, Iorio A, Bazzan M, Moia M, Guazzaloca G, Bertoldi A, Tomasi C, Scannapieco G, Ageno W: Three months versus one year of oral anticoagulant therapy for idiopathic deep venous thrombosis. Warfarin Optimal Duration Italian Trial Investigators. N Engl J Med 2001; 345:165-169. 